\title{
Recent developments in particle yield fluctuation measurements
}

\author{
Igor Altsybeev ${ }^{1, *}$ \\ ${ }^{1}$ Saint-Petersburg State University, Saint Petersburg, Russia
}

\begin{abstract}
In relativistic heavy-ion collisions, properties of the initial state and effects arising during evolution of the medium, such as a transition between the hadronic and partonic phases, should reflect themselves in event-by-event fluctuations of the number of produced particles. In this paper, recent measurements of several event-by-event observables, namely, dynamical fluctuations of relative particle yields and forwardbackward correlations of different types, are discussed. Also, new observables for forward-backward correlation studies are proposed: correlations between ratios of identified particle yields in two separated acceptance intervals and the correlation between the ratio in one interval and average transverse momentum in another.
\end{abstract}

\section{Introduction}

Measurement of the properties of dense nuclear matter and search for signs of its transition to the state of deconfinement are performed by studying collisions between heavy ions. One way to probe the phase structure of strongly interacting matter is the analysis of particle production in the framework of the statistical hadronization model, which accounts for the thermal distribution of particle species. At the LHC energies, where baryo-chemical potential is close to zero, results of this model can be directly compared with the lattice QCD calculations. The value of the pseudo-critical temperature extracted from data is $T_{c}=156.2 \pm 1.5 \mathrm{MeV}$ [1], which corresponds to the lattice results.

Another approach to the search for critical behavior in hadronic matter is analysis of fluctuations of various quantities. Examples of such quantities are the conserved charges, in particular, the residual electric charge, baryon number and strangeness. Event-by-event fluctuations help to characterize the properties of the "bulk" of the system and also are closely related to dynamics of the phase transitions. A non-monotonic behaviour with experimentally varied parameters such as the collision energy, centrality, system size, rapidity is expected [2].

In comparison with the "event-averaged" observables (like average particle yields, spectra), observables based on fluctuations are much more sensitive to different biases which can distort the results: limited detector acceptance, non-flat particle registration efficiency and its dependence on detector occupancy, contamination by secondary particles, mis-identification of particle species, conservation laws, resonance decays, and, finally, trivial event-by-event fluctuations of collision geometry (so-called "volume fluctuations"). From this long list of caveats it becomes evident, that for this kind of studies it is not enough just to define a new observable - it's essential to know how robust this observable is in a real experiment, and a correction procedure must be provided, if necessary.

In this article, properties and experimental measurements of some fluctuation-based observables are reviewed. After that, new fluctuation observables based on particle number ratios are introduced.

\footnotetext{
*e-mail: i.altsybeev@spbu.ru
} 


\section{Robust observables for fluctuation measurements}

Particle number fluctuations can be quantified by different measures. The simplest one is the variance of multiplicity distribution, however, it depends on the volume of the system and its fluctuations (an extensive observable), which makes it tricky to extract the "dynamical" contribution, in which we are really interested in. Within the grand canonical ensemble, the volume cancels if the variance is scaled with the mean multiplicity (an intensive observable), but the dependence on volume fluctuations still remains.

Fluctuations can be studied also in terms of variations in the ratio of particle yields of two distinct species. Denote number of particles of type $a$ and $b$, measured in a given event, as $n_{a}$ and $n_{b}$, then the ratio of the two yields is $r=n_{a} / n_{b}$. The quantity we are interested in is the variance of this ratio, $\left\langle\Delta r^{2}\right\rangle$. If one normalizes this quantity to the mean and assumes that deviations of the yields from the means $n_{a}-\left\langle n_{b}\right\rangle$ and $n_{a}-\left\langle n_{b}\right\rangle$ are small, then the following approximation is valid:

$$
v \equiv \frac{\left\langle\Delta r^{2}\right\rangle}{\langle r\rangle^{2}} \approx\left\langle\left(\frac{n_{a}}{\left\langle n_{a}\right\rangle}-\frac{n_{b}}{\left\langle n_{b}\right\rangle}\right)^{2}\right\rangle \text {. }
$$

In the limit of independent particle production (Poisson statistics), this quantity is reduced to just $v_{\text {stat }}=1 /\left\langle n_{a}\right\rangle+1 /\left\langle n_{b}\right\rangle$. In experimental studies, this trivial contribution is usually subtracted from the value of $v$, so the observable which is actually measured is [3]

$$
v_{d y n} \equiv v-v_{\text {stat }}=\frac{\left\langle n_{a}\left(n_{a}-1\right)\right\rangle}{\left\langle n_{a}\right\rangle^{2}}+\frac{\left\langle n_{b}\left(n_{b}-1\right)\right\rangle}{\left\langle n_{b}\right\rangle^{2}}-2 \frac{\left\langle n_{a} n_{b}\right\rangle}{\left\langle n_{a}\right\rangle\left\langle n_{b}\right\rangle} .
$$

The value of $v_{d y n}$ is non-zero when production of particles $A$ and $B$ is correlated (non-Poissonian). This observable is robust against efficiency losses and volume fluctuations. It was used to measure net-charge fluctuations in a number of experiments, as well as relative particle yield fluctuations $(\mathrm{K} / \pi, \mathrm{p} / \pi$ and $\mathrm{K} / \mathrm{p})$, for instance, by ALICE [4]. It was pointed, that for interpretation of the results, obtained with $v_{d y n}$, the acceptance coverage is crucial, and also that resonance contributions should be better understood before making conclusions about dynamical effects. It should be stressed that measurements of $v_{d y n}$ are typically performed within one single pseudorapidity window, in most cases this is actually a full $\eta$-acceptance of a given experiment.

A class of observables which depend neither on the volume fluctuations of the system nor on the volume itself (the so-called strongly intensive quantities) was introduced in [5]. In fact, one of these observables, denoted as $\Sigma$, is closely related to $v_{d y n}$ - the difference is only in the scaling factor proportional to multiplicity densities, which are easy to correct for detector efficiency. In the model of independent sources, the $\Sigma$ quantity measures the properties of a single source [6]. For Poissonian particle production, $\Sigma=1$. Recently, the $\Sigma$ observable was used to quantify the strength of the forward-backward multiplicity correlations of charged particles between the two separated $\eta$ intervals in $\mathrm{Pb}-\mathrm{Pb}$ collisions $\sqrt{s_{\mathrm{NN}}}=2.76 \mathrm{TeV}$ [7]. Usage of this observable allows one to eliminate the big problem of the forward-backward correlation studies - the contribution from the volume fluctuations. Peculiar change of $\Sigma$ with centrality was obtained, which is not understood so far.

Another type of forward-backward observable which is robust against the volume fluctuations is a correlation between the mean transverse momenta measured event-by-event in the two $\eta$-windows. Non-trivial centrality evolution of the mean- $p_{\mathrm{T}}$ correlation strength has been recently obtained in $\mathrm{Pb}-\mathrm{Pb}$ collisions in ALICE [8].

\section{Correlations between ratios of particle yields}

In this section, a new type of observables for correlation studies is introduced, namely, a correlation between the particle number ratios measured in two separated rapidity intervals. Note that we can straightforwardly add splitting of the acceptance intervals also in other dimensions (in $\varphi$ and in $\left.p_{\mathrm{T}}\right)$. The correlation coefficient between particle number ratios can be written as

$$
b_{\text {corr }}=\frac{\left\langle r^{F} \cdot r^{B}\right\rangle}{\left\langle r^{F}\right\rangle\left\langle r^{B}\right\rangle}-1,
$$


where $r^{F}=n_{a}^{F} / n_{b}^{F}$ and $r^{B}=n_{a}^{B} / n_{b}^{B}$ are ratios of multiplicities for particles of species $a$ and $b$ measured in the two acceptance windows (let us call them "forward" and "backward"), and angular brackets denote averaging over events. For example, we can consider a ratio of a number of kaons to a number of pions, $r=n_{K} / n_{\pi}$, in this case the correlator in (3) becomes $\left\langle r^{F} \cdot r^{B}\right\rangle=\left\langle n_{\mathrm{K}}^{F} / n_{\pi}^{F} \cdot n_{\mathrm{K}}^{B} / n_{\pi}^{B}\right\rangle$.

When multiplicities of produced particles are large (in semi-central and central A-A collisions), the event-by-event fluctuations of them are expected to be small relative to the average values. In this case, it can be shown that the following approximation for the $b_{\text {corr }}$ is valid:

$$
b_{\text {corr }} \approx \frac{\left\langle n_{a}^{F} n_{a}^{B}\right\rangle}{\left\langle n_{a}^{F}\right\rangle\left\langle n_{a}^{B}\right\rangle}+\frac{\left\langle n_{b}^{F} n_{b}^{B}\right\rangle}{\left\langle n_{b}^{F}\right\rangle\left\langle n_{b}^{B}\right\rangle}-\frac{\left\langle n_{a}^{F} n_{b}^{B}\right\rangle}{\left\langle n_{a}^{F}\right\rangle\left\langle n_{b}^{B}\right\rangle}-\frac{\left\langle n_{b}^{F} n_{a}^{B}\right\rangle}{\left\langle n_{b}^{F}\right\rangle\left\langle n_{a}^{B}\right\rangle} \stackrel{\text { def. }}{\equiv} v_{\mathrm{FB}}^{a / b} .
$$

Each term in this expression is a normalized correlator between multiplicities in forward and backward intervals. If we adopt here the notion of the normalized cumulants $R_{2}^{a b}=\left\langle n_{a}^{F} n_{b}^{B}\right\rangle /\left\langle n_{a}^{F}\right\rangle\left\langle n_{b}^{B}\right\rangle-1$, the expression (4) can be rewritten as

$$
v_{\mathrm{FB}}^{a / b}=R_{2}^{a a}+R_{2}^{b b}-R_{2}^{a b}-R_{2}^{b a} .
$$

In case of independent (Poissonian) particle production $v_{\mathrm{FB}}=0$. It is zero also if only short-range correlations are present in the system, and a separation between the two observation windows is large enough to suppress them. Another important property of $v_{\mathrm{FB}}$ is its robustness to volume fluctuations as well as to efficiency of particle detection (if the efficiency is constant within the acceptance windows). In the model with independent particle-emitting sources, the scaling of this observable with the average number of sources $\left\langle N_{\mathrm{s}}\right\rangle$ is $v_{\mathrm{FB}}=1 /\left\langle N_{\mathrm{s}}\right\rangle \cdot v_{\mathrm{FB}}^{\mathrm{s}}$, where $v_{\mathrm{FB}}^{\mathrm{s}}$ is the value of this quantity for a single source. In fact, the properties of the $v_{\mathrm{FB}}$ observable mentioned above are similar to those of the $v_{\text {dyn }}$ (2) [3]. It is important to note also, that it's possible to correct individual terms of $v_{\mathrm{FB}}$ (moments of the first and the second orders) for particle mis-identification by applying the Identity Method [9].

The definition of $v_{\mathrm{FB}}$ is also closely connected to observables used for the charge-dependent correlation studies: if one takes for the analysis the ratios of multiplicities of positively and negatively charged particles (i.e. $r^{F}=n_{+}^{F} / n_{-}^{F}$ and $r^{B}=n_{+}^{B} / n_{-}^{B}$ ), then (5) reads as $v_{\mathrm{FB}}^{+/-}=R_{2}^{++}+R_{2}^{--}-R_{2}^{+-}-R_{2}^{-+}$, and the relation to the charge-dependent correlation function $R_{\mathrm{CD}}$, adopted, for example, in [10], is $\nu_{\mathrm{FB}}=-4 \cdot R_{\mathrm{CD}}$. Note that this relation between observables can be treated in the opposite way: in case of large enough multiplicities in $F$ and $B$ acceptance windows, the $R_{\mathrm{CD}}$ observable for charge-dependent correlations is nothing else than the approximation for the correlation coefficient $b_{\text {corr }}$ between particle number ratios (3), multiplied by $-1 / 4$. The connection to the balance function observable is also straightforward: $b . f .=-\rho_{1}^{c h} / 4 \cdot v_{\mathrm{FB}}^{+/-}$, where $b . f$. is the balance function between $F$ and $B$ windows and $\rho_{1}^{c h}$ is a charged particle density.

Consider the particular case for the $b_{\text {corr }}$ observable, when the ratio between number of kaons to number of pions is taken: $r^{F}=n_{\mathrm{K}}^{F} / n_{\pi}^{F}$ and $r^{B}=n_{\mathrm{K}}^{B} / n_{\pi}^{B}$, then

$$
b_{\mathrm{corr}}^{\mathrm{K} / \pi} \approx \nu_{\mathrm{FB}}^{\mathrm{K} / \pi}=R_{2}^{\mathrm{KK}}+R_{2}^{\pi \pi}-R_{2}^{\mathrm{K} \pi}-R_{2}^{\pi \mathrm{K}} .
$$

With this observable, a correlation between strangeness production at large $\eta$ gaps can be probed - the physics case of interest for thermal models [1], models with interactions between color ropes (quark-gluon strings) [11, 12], etc.

Values of the correlation strength $b_{\text {corr }}^{\mathrm{K} / \pi}$ were calculated in $\mathrm{Pb}-\mathrm{Pb}$ collisions at $\sqrt{S_{\mathrm{NN}}}=2.76 \mathrm{TeV}$ in AMPT and HIJING event generators. AMPT version with the string melting and rescattering options turned on was used. On the left panel in Fig. 1, centrality dependence of the $b_{\text {corr }}$ calculated by the direct formula (3) is shown in red squares. Values are multiplied by charge particle density $\left\langle\mathrm{d} N_{\mathrm{ch}} / \mathrm{d} \eta\right\rangle$ in order to cancel out the $1 /\left\langle N_{\mathrm{s}}\right\rangle$ scaling of this observable mentioned earlier. Scaled values of $\nu_{\mathrm{FB}}$ given by the approximation (6) are shown as blue circles, it can be seen that they are 

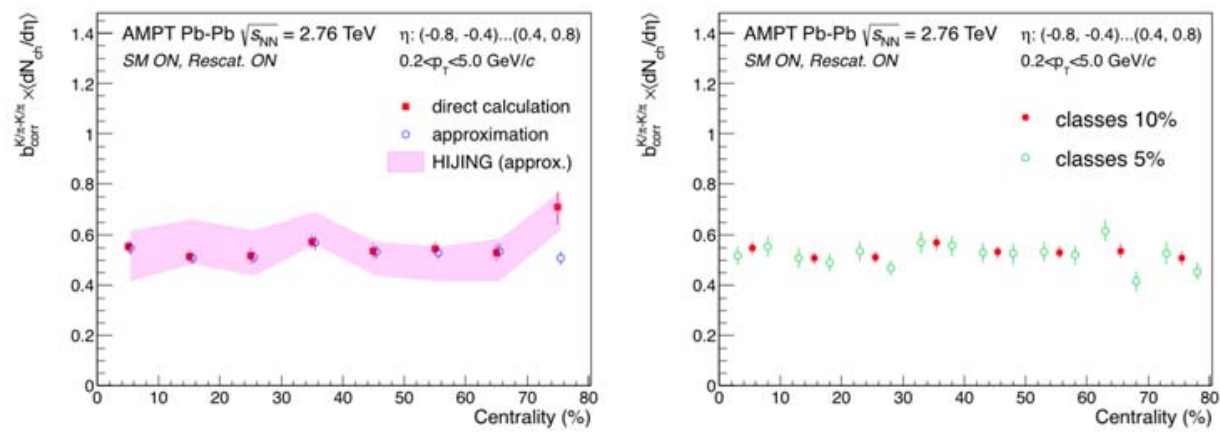

Figure 1. Scaled strength of forward-backward correlations between $n_{\mathrm{K}} / n_{\pi}$ ratios as a function of centrality in $\mathrm{Pb}-\mathrm{Pb}$ collisions at $\sqrt{s_{\mathrm{NN}}}=2.76 \mathrm{TeV}$. Forward-backward $\eta$-intervals are $(-0.8,-0.4)$ and $(0.4,0.8), p_{\mathrm{T}}$ range of particles is $0.2-5.0 \mathrm{GeV} / c$. Left: direct calculations in AMPT (full squares) in comparison with the approximation (open circles). Results from HIJING are shown as a filled band. Right: correlation strength in centrality classes of widths $10 \%$ and $5 \%$ (classes are determined using charge particle multiplicities at forward $\eta$-ranges corresponding to the $\mathrm{V} 0$ detector of ALICE)

in agreement with the direct evaluation of $b_{\text {corr }}$. Centrality dependence of the correlation strength is flat in AMPT, similarly to HIJING (shown on the same plot as a shaded band), and the correlation strength is positive. The right panel of the Fig. 1 demonstrates robustness of this quantity to the volume fluctuations: values of $b_{\text {corr }}$ are the same for centrality classes of different width.

The same analysis can be performed with the additional splitting of the $\eta$-windows into azimuthal sectors. Left panel of Fig. 2 shows dependence of $b_{\text {corr }}$ on the distance $\varphi_{\text {sep }}$ between the two azimuthal windows in $\mathrm{Pb}-\mathrm{Pb}$ collisions in $10-20 \%$ centrality class in AMPT. The approximate expression $\nu_{\mathrm{FB}}$ works well even in this case, when particle multiplicities in the acceptance windows are quite low (in each $\varphi$-window here, $\left\langle n_{\pi}\right\rangle \approx 13,\left\langle n_{\mathrm{K}}\right\rangle \approx 1.5$ ). The "near-side" peak at $\left|\varphi_{\text {sep }}\right|<\pi / 2$ is observed, while $b_{\text {corr }}$ is close to 0 at $\varphi_{\text {sep }} \approx \pi$. As for HIJING, results seem to be consistent with a flat dependence on $\varphi_{\text {sep }}$, however, statistical uncertainties are quite large to make a definite statement.
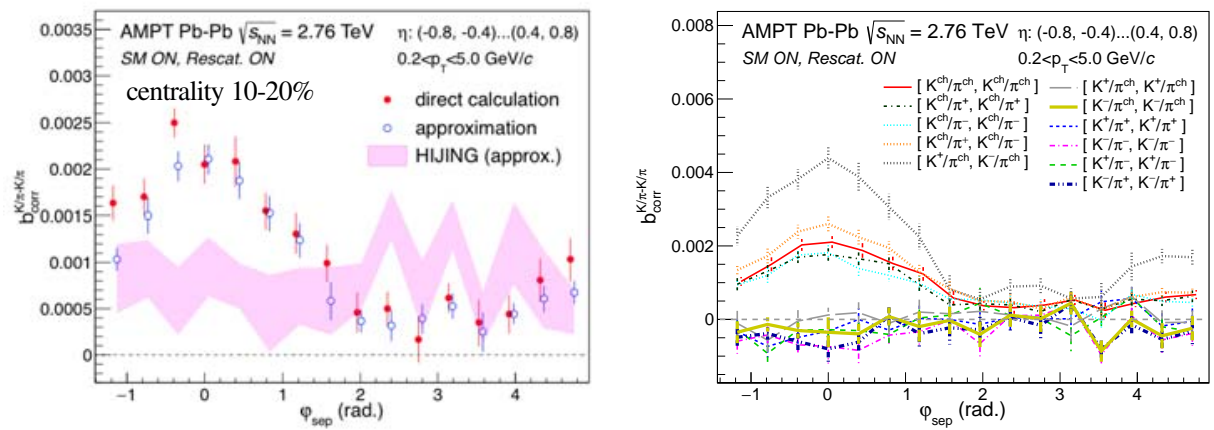

Figure 2. Forward-backward correlation strength $b_{\text {corr }}^{\mathrm{K} / \pi}$ as a function of the distance $\varphi_{\text {sep }}$ between the two azimuthal windows in 10-20\% centrality class of $\mathrm{Pb}-\mathrm{Pb}$ collisions in AMPT. Left: direct calculations in AMPT (full circles) in comparison with the approximation (open circles). Results from HIJING are shown as a filled band. Right: correlation strength for different charge combinations of kaons and pions in the two windows 
Obviously, the correlation strength is affected by different contributions not directly related to the "collective effects" we would like to access. The main "non-dynamical" contributions are resonance decays and conservation laws. For example, in case of $\mathrm{K} / \pi$ analysis, the strangeness conservation provides correlations which can be seen in the right panel of the Fig. 2: the solid red line shows results for all charged kaons and pions measured in each window, while other lines correspond to more differential results, when only certain charge combinations are used. It can be seen, that when kaons of the opposite sign are counted in both forward and backward window (this corresponds to the first column in the legend for this plot), the correlation strength is significant. The largest correlation, in fact, is for the case when one window counts only $\mathrm{K}^{+}$and another - only $\mathrm{K}^{-}$(upper gray curve). On the other hand, if only kaons of a particular charge sign are counted in both windows (right column in the legend), the correlation strength is nearly zero.

\section{Correlations between particle ratio and mean transverse momentum}

Here we introduce another potentially useful observable, namely, a correlation between the particle yield ratio in one acceptance window and the mean transverse momentum of particles in another:

$$
b_{\mathrm{corr}}^{\bar{p}-r}=\frac{\left\langle\bar{p}^{F} \cdot r^{B}\right\rangle}{\left\langle\bar{p}^{F}\right\rangle\left\langle r^{B}\right\rangle}-1,
$$

where $\bar{p} \equiv \sum_{i=1}^{n} p_{\mathrm{T}}^{i} / n$ is the mean transverse momentum of particles in a given event within acceptance cuts. In case of modest event-by-event fluctuations of particle multiplicities with respect to their means, the approximate expression for this observable is

$$
b_{\mathrm{corr}}^{\bar{p}-r} \approx \frac{\left\langle\bar{p}^{F} \cdot n_{\mathrm{K}}^{B}\right\rangle}{\left\langle\bar{p}^{F}\right\rangle\left\langle n_{\mathrm{K}}^{B}\right\rangle}-\frac{\left\langle\bar{p}^{F} \cdot n_{\pi}^{B}\right\rangle}{\left\langle\bar{p}^{F}\right\rangle\left\langle n_{\pi}^{B}\right\rangle} \stackrel{\text { def. }}{=} v_{\mathrm{FB}}^{\bar{p}-r} .
$$

Properties of this quantity, namely, scaling with a number of particle sources and robustness to efficiency and volume fluctuations, are similar to the $v_{\mathrm{FB}}$ introduced above. With this observable, one can study, for instance, correlations between a relative number of strange particles in one rapidity interval and the density of the fireball (which reflects itself in $\bar{p}$ ) in another.

Let us take for the backward window the ratio between the number of kaons to the number of pions, as before. Left panel of Fig. 3 shows centrality dependence of the $b_{\mathrm{corr}}^{\bar{p}-\mathrm{K} / \pi}$ calculated in
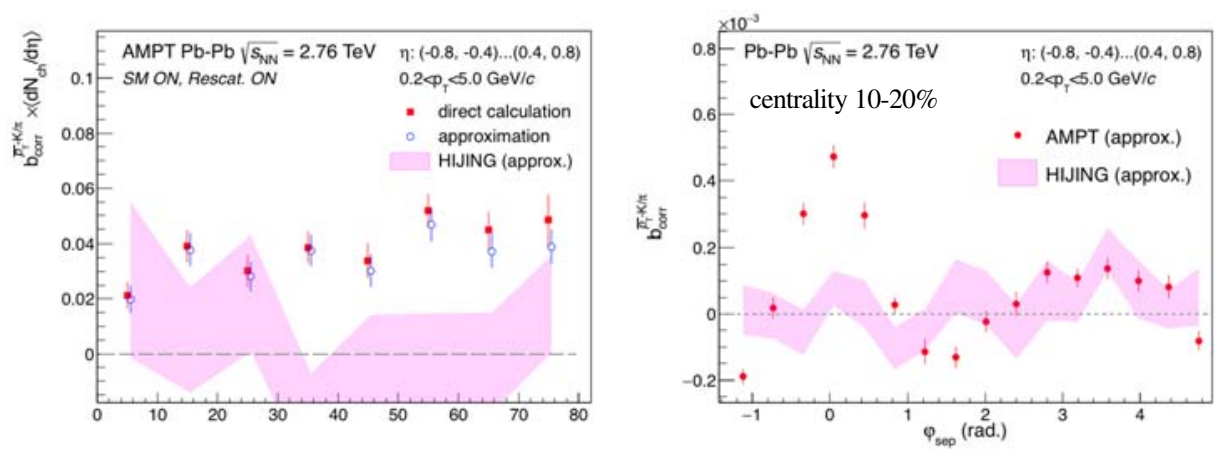

Figure 3. Forward-backward correlation strength $b_{\text {corr }}^{\bar{p}-\mathrm{K} / \pi}$ in $\mathrm{Pb}-\mathrm{Pb}$ collisions in AMPT as a function of collision centrality, scaled by charged particle density (left panel), and as a function of the distance $\varphi_{\text {sep }}$ between the two azimuthal windows in 10-20\% centrality class (right panel). Results from HIJING are shown as filled bands. Kinematic ranges are indicated on the plots 
AMPT for the pair of $\eta$-windows, scaled with the multiplicity density. The same plot demonstrates also the agreement between the approximate expression (8) and the direct calculations. On the right panel, results of the analysis in azimuthal intervals are shown as a function of $\varphi_{\text {sep }}$ (analogously to the Fig. 2). There is a peak at the near-side, and also some away-side structure is observed. On both plots, values of $b_{\text {corr }}^{\bar{p}-\mathrm{K} / \pi}$ in HIJING are consistent with zero, which is in line with the absence of collective effects in this event generator.

Non-zero values of $b_{\text {corr }}^{\bar{p}-r}$ may provide new information about the collective effects in the medium. In order to further suppress contributions from resonances and conservation laws, one could, in addition to the large $\eta$-gap between the windows, take for the ratio and mean $p_{\mathrm{T}}$ only the same-charge particles, or differentiate the analysis in other ways, for example, calculate $\bar{p}$ only for particles of a particular species.

\section{Summary and outlook}

In this article, we have briefly reviewed a set of fluctuation-based observables which possess useful properties like robustness against limited particle registration efficiency and fluctuations of the collision geometry, and mentioned some recent experimental measurements with these quantities in A-A collisions over the passed few years.

In the second part of the paper, new observables for fluctuation studies have beed introduced. The first type of the proposed observables quantifies the correlation between ratios of identified particle yields measured in two separated acceptance bins, the second type - the correlation between the ratio in one bin and average transverse momentum in the other. With these observables it is possible, for instance, to study a correlation between relative strangeness yield in one rapidity interval and the density of the fireball, formed in A-A collisions, in another interval. Approximate expressions, which reveal a connection between the correlation strengths and a sum of normalized cumulants, have been provided. It is shown that these measures are also immune to volume fluctuations and detector inefficiencies. The problem of particle mis-identification for these observables can be solved by utilizing the Identity Method. Evolution of the proposed quantities with centrality of A-A collisions has been calculated in AMPT and HIJING event generators. Behaviour of these quantities in other models is to be investigated.

Acknowledgements. This work is supported by the Russian Science Foundation, grant 17-7220045.

\section{References}

[1] A. Andronic et al., Nature 561, 321-330 (2018)

[2] M. Stephanov, K. Rajagopal, E. Shuryak, Phys. Rev. D 60, 114028 (1999)

[3] C. Pruneau, S. Voloshin, S. Gavin, Phys. Rev. C 66, 044904 (2002)

[4] ALICE Collaboration, arXiv:1712.07929, CERN-EP-2017-318 (2017)

[5] M. Gorenstein, M. Gazdzicki, Phys. Rev. C 84, 014904 (2011)

[6] E. Andronov, V. Vechernin, Eur. Phys. J. A 55(1), 14 (2019)

[7] I. Sputowska, ALICE Collaboration, talk at the conference "Hot Quarks", September 7-14, 2018

[8] I. Altsybeev, KnE Energy and Physics 3(1), 304-312 (2018)

[9] M. Gazdzicki et al., Phys. Rev. C 83, 054907 (2011)

[10] ALICE Collaboration, arXiv:1805.04422, CERN-EP-2018-118 (2018)

[11] V. Kovalenko, V. Vechernin, J. Phys.: Conf. Ser. 668, 012065 (2016)

[12] C. Bierlich, EPJ Web of Conferences 171, 14003 (2018) 